\title{
Micro-solvent Cluster Extraction Using Aqueous Mixed Solvents of Ionic Liquid
}

\author{
Thiraporn Charoenraks, Masaaki TABata, ${ }^{\dagger}$ and Kenta FuJII \\ Department of Chemistry, Faculty of Science and Engineering, Saga University, \\ 1 Honjo-machi, Saga 840-8502, Japan
}

\begin{abstract}
Organic compounds (2-naphthol, phenol, 4-chlorophenol, 4-nitrophenol, and 1,3,5-naphthalenetrisulfonic acid) were sufficiently separated from mixtures during flow in a fused silica capillary tube $(50 \mu \mathrm{m}$ in i.d. and $45 \mathrm{~cm}$ in length) with an aqueous mixed solvent of an ionic liquid, 1-butyl-3-methylimidazolium chloride $\left(\mathrm{BMIM}^{+} \mathrm{Cl}^{-}\right)$, without a specific separation column. The method is based on micro-solvent cluster formation in aqueous mixed solvents of ionic liquid and preferential solvation of solvent clusters to analytes. The measurement of large angle X-ray scattering (LAXS) of aqueous mixed solvents with an ionic liquid of tetrafluoroborate $\left(\mathrm{BMIM}^{+} \mathrm{BF}_{4}^{-}\right)$indicated the formation of micro-solvent clusters of water and ionic liquid in the mixed solvent. A neutral polymer (polyvinylpyrrolidone, PVP) enhanced the separation. Polarized or ionic molecules eluted slowly. The theoretical plate numbers were 6320, 22907, 63645, and 37184 for 2naphthol, phenol, 4-chlorophenol, and 4-nitrophenol, respectively, under the conditions of $1.0 \mathrm{M}$ of $\mathrm{BMIM}^{+} \mathrm{Cl}^{-}$and $0.1 \mathrm{M}^{-}$ of PVP; the flow rate was $1 \mu \mathrm{L} \mathrm{min}$. The separation mechanism is discussed from the viewpoint of the partition of analytes between micro-solvent clusters of water and organic solvent molecules.
\end{abstract}

(Received April 2, 2008; Accepted May 22, 2008; Published October 10, 2008)

\section{Introduction}

The separation method is very important and essential for not only analytical chemistry, but also other fields, such as environment sciences, organic synthesis, biology, pharmacology, medicine, and industries. Among the separation methods, high performance liquid chromatography (HPLC) ${ }^{1,2}$ has been widely used in these fields, and many separation columns are available for analysis. The inner composition of separation columns, however, is not clear at all, due to patents. In this paper, a new separation method without specific separation columns is described, which optimizes the separation conditions by choosing watersoluble organic solvents and their composition for analysis.

The separation principle is micro-solvent cluster formation in mixed solvents ${ }^{3-6}$ and preferential solvation of the solvent molecules to analytes in the mixed solvents. ${ }^{7,8}$ Aqueous mixed solvents, like the mixture of acetonitrile and water, are mixed homogeneously on the macro scale, though the mixtures are heterogeneous on the micro scale. The microheterogeneity was directly observed by a small-angle neutron-scattering method (SANS) for mixtures of water and acetonitrile, tetrahydrofuran and 1,4-dioxane. ${ }^{9}$ The SANS data gave clear evidence for solvent cluster formation, where water molecules associate with other water molecules and acetonitrile molecules associate with other acetonitrile molecules to form solvent clusters of water and acetonitrile, respectively. The microheterogeneity increased upon the addition of $\mathrm{NaCl}^{6}{ }^{6}$ In addition, preferential solvation occurs to chemical species dissolved in aqueous mixed solvents. This phenomenon was confirmed by measuring the change in

$\dagger$ To whom correspondence should be addressed.

E-mail: tabatam@cc.saga-u.ac.jp the fluorescence spectrum. ${ }^{8}$ Compounds that are soluble in water are solvated preferentially by water molecules, and those that are soluble in organic solvents are solvated preferentially by organic solvent molecules. Due to the characteristics of the mixed solvents described above, i.e. micro-solvent cluster formation and preferential solvation, compounds dissolved in mixed solvents are "extracted" to their preferred solvent clusters, water or organic solvent clusters. The partition of the compounds between two solvent clusters depends on their chemical and physical properties. Therefore, the compounds dissolved in mixed solvents are spontaneously separated in mixed solvents. This idea has been explored to concerning the separation of chemical species using mixed solvents and a capillary without any specific separation column. ${ }^{10}$ Compounds injected to a capillary are separated during their flow in a capillary tube and eluted along with the difference in solubility of the compounds in micro-solvent clusters.

In this study, an aqueous mixed solvent of an ionic liquid (IL), 1-butyl-3-methylimidazolium chloride $\left(\mathrm{BMIM}^{+} \mathrm{Cl}^{-}\right)$, was used to separate of model compounds (2-naphthol, phenol, 4chlorophenol, 4-nitrophenol, and 1,3,5-naphthalenetrisulfonic acid) using an open tubular capillary. Ionic liquids are suitable solvents as mobile phase solutions when carrying out separation by a capillary tube, because of their miscibility with water at any ratio, as well as their unique properties, such as low vapor pressure, viscosity, hydrophobicity, and thermal and chemical stabilities. ${ }^{11}$

To confirm the formation of micro-solvent clusters in mixed solvents, the measurement of large angle X-ray scattering (LAXS) for aqueous mixed solvents with an ionic liquid of tetrafluoroborate $\left(\mathrm{BMIM}^{+} \mathrm{BF}_{4}^{-}\right)$was carried out at different volume fractions of the ionic liquid $(1.0,0.7,0.5,0.3$, and $0(\mathrm{v} / \mathrm{v}))$. 


\section{Experimental}

Reagents

An ionic liquid, 1-butyl-3-methylimidazolium chloride $\left(\mathrm{BMIM}^{+} \mathrm{Cl}^{-}\right)$, was synthesized according to literature procedures. $\mathrm{BMIM}^{+} \mathrm{Cl}^{-}$is a solid, but is very soluble in water. Its mixed aqueous solvents were prepared by weighing $\mathrm{BMIM}^{+} \mathrm{Cl}^{-}$and dissolving then it in water at a given ratio. Polyvinylpyrrolidone (PVP), 2-naphthol, phenol, 4-chlorophenol, 4-nitrophenol, and 1,3,5-naphthalenetrisulfonic acid were purchased from Wako Pure Chemical Industry Co., Ltd. Japan. Double-distilled water was used throughout. All reagents were of analytical reagent grade.

\section{Apparatus}

The separation system consisted of a syringe driver (Syringe Pump Controller MF-9090 with an adjustable flow rate at 0.1 $\mu \mathrm{L}\left(\mathrm{L}=\mathrm{dm}^{3}\right) \mathrm{min}^{-1}$ to $100 \mu \mathrm{L} \mathrm{min}{ }^{-1}$, BAS, USA) together with a Hamilton Gastight $1 \mathrm{~mL}\left(\mathrm{~mL}=\mathrm{cm}^{3}\right)$ syringe for pumping the mobile-phase solution. A $0.5 \mu \mathrm{L}$ micro-sample injector (Model 7520, Rheodyne, USA) linked with a homemade "T" splitter that can reduce the injected sample volume was used for sample injection. The sample solution flowed through a fused-silica capillary tube $(50 \mu \mathrm{m}$ and $375 \mu \mathrm{m}$ in i.d. and o.d., respectively, and $45 \mathrm{~cm}$ in length) from the injection port to the detection window. An intelligent UV/Vis detector of capillary (CE-970, JASCO, Japan) was used for detection of the eluted compounds. The detector was connected to a personal computer through JASCO LC-NET. Data collection and chromatogram analysis were accomplished by JASCO-Borwin ${ }^{\circledR}$. The concentrations of analytes were $2.5 \times 10^{-3} \mathrm{M}\left(\mathrm{M}=\mathrm{mol} \mathrm{dm}^{-3}\right)$.

\section{Separation method}

Mixed solvents of water and ionic liquid of $\mathrm{BMIM}^{+} \mathrm{Cl}^{-}$were used as the mobile phase at various concentrations of $\mathrm{BMIM}^{+} \mathrm{Cl}^{-}$. Stock solutions of 2-naphthol, phenol, 4-chlorophenol, 4nitrophenol, and 1,3,5-naphthalenetrisulfonic acid were prepared by dissolving each compound in a small amount of methanol. A mixture of 2-naphthol, phenol, 4-chlorophenol, 4-nitrophenol, and 1,3,5-naphthalenetrisulfonic acid was prepared by the addition of each stock solution to a mixed solvent containing water and $\mathrm{BMIM}^{+} \mathrm{Cl}^{-}$and by dilution to $2.5 \mathrm{mM}$ with the mixed solvents. All chromatograms were obtained at room temperature controlled at $25^{\circ} \mathrm{C}$ and at a flow rate of $1 \mu \mathrm{L} \mathrm{min}$. The void volume, $v_{0}\left(=t_{0}\right)$, of the capillary was determined by using methanol. The compounds were detected at $223 \mathrm{~nm}$. Before injecting the samples, the capillary was washed with $0.1 \mathrm{M}$ $\mathrm{NaOH}$ for $30 \mathrm{~min}$, followed by water for $30 \mathrm{~min}$. For each use of different concentrations of ionic liquid, the capillary was washed every time with water and $0.1 \mathrm{M} \mathrm{NaOH}$ for $30 \mathrm{~min}$, respectively, followed by water for $60 \mathrm{~min}$ to confirm a completely same experimental condition in the capillary tube.

\section{LAXS measurement}

High-energy X-ray scattering measurements were carried out using the BL04B2 beam-line of SPring-8 at Japan Synchrotron Radiation Research Institute (JASRI). ${ }^{12,13}$ Monochromated Xrays of $61.7 \mathrm{keV}$ were obtained using a $\mathrm{Si}$ (220) monochromator. The observed X-ray intensity was corrected for absorption, polarization, and incoherent scatterings to obtain coherent scatterings, $I_{\mathrm{coh}}(s){ }^{14,15}$ The detailed procedure of data analysis was described before. ${ }^{13,16}$ The structure function, $i(s)$, and the correlation function, $g(r)$, were obtained from

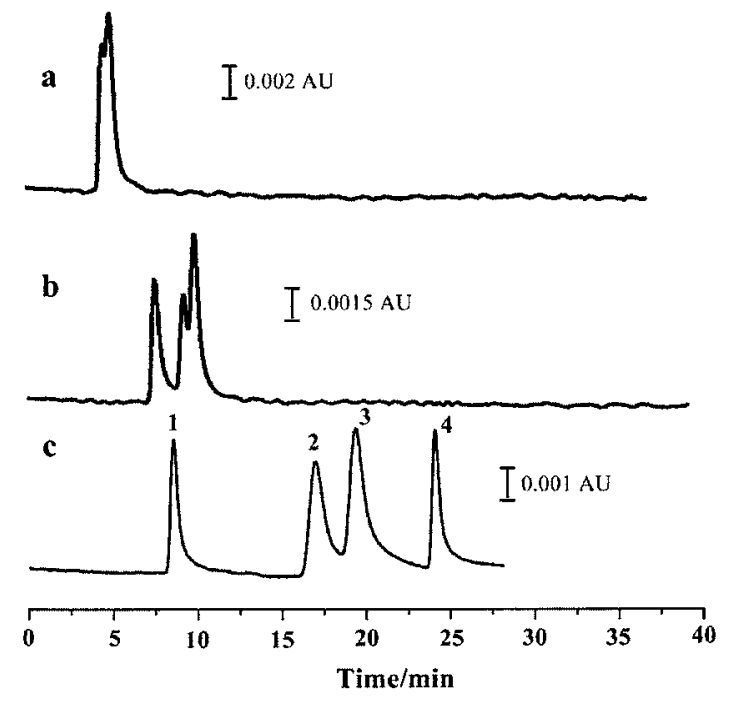

Fig. 1 Chromatograms of $2.5 \mathrm{mM}$ phenol derivatives with a mobile phase containing different concentrations of $\mathrm{BMIM}^{+} \mathrm{Cl}^{-}$: (a) 0.5 , (b) 0.8 , (c)1.0 M. Chromatographic conditions: flow rate, $1 \mu \mathrm{L} \mathrm{min} \mathrm{m}^{-1}$, detection, $223 \mathrm{~nm}$; room temperature. Peaks: (1) 2-naphthol, (2) phenol, (3) 4-chlorophenol, (4) 4-nitrophenol.

$$
\begin{aligned}
& i(s)=\frac{I_{\operatorname{coh}}(s)-\sum n_{\mathrm{i}} f_{\mathrm{i}}^{2}(s)}{\left(\sum n_{i} f_{\mathrm{i}}(s)\right)^{2}}, \\
& g(r)=1+\frac{1}{2 \pi^{2} r \rho_{0}} \int_{0}^{s} s i(s) \sin (r s) \exp \left(-B s^{2}\right) \mathrm{d} s,
\end{aligned}
$$

where $n_{\mathrm{i}}$ and $f_{\mathrm{i}}(s)$ denote the number and the atomic scattering factor of atom $i$, respectively; ${ }^{17} \rho_{0}$ is the number density, and $B$ is the damping factor $\left(0.01 \AA^{2}\right.$ in this work). KURVLR was used for the X-ray scattering data treatment and analysis. ${ }^{18}$ The detailed procedure has been described previously. ${ }^{16}$

\section{Results and Discussion}

Figure 1 shows a chromatogram of a mixture of four phenol derivatives: (2-naphthol, phenol, 4-chlorophenol, and 4nitrophenol) at the different concentrations of $\mathrm{BMIM}^{+} \mathrm{Cl}^{-}$. The separation of the compounds depended on the concentration of $\mathrm{BMIM}^{+} \mathrm{Cl}^{-}$. The mixture was separated at concentrations higher than $1.0 \mathrm{M}^{\text {of }} \mathrm{BMIM}^{+} \mathrm{Cl}^{-}$and eluted in the order of 2-naphthol, phenol, 4-chlorophenol, and 4-nitrophenol. The elution time of the compounds was confirmed by injecting each compound separately. Polar and acidic compounds eluted slowly. The $\mathrm{p} K_{\mathrm{a}}$ values of the phenol derivatives are 9.46 (2-naphthol), 9.95 (phenol), 9.38 (4-chlorophenol), 7.08 (4-nitrophenol), respectively. ${ }^{19}$ The $\mathrm{p} K_{\mathrm{a}}$ value of 2-naphthol is lower than that of phenol, but it eluted faster than phenol. This result suggest that the hydrophobicity of the compounds governs the elution order more than $\mathrm{p} K_{\mathrm{a}}$ of the compound in the present system. Watersoluble compounds are retained in water-clusters on the surface of the capillary tube, and non-polar compounds are concentrated at the center of the capillary tube, resulting in the abovementioned elution order. The proposed elution mechanism is discussed in Separation mechanism below.

Neutral organic compounds were successfully separated by injection and flow through a capillary tube with the mobile phase of a mixed solvent of water and $\mathrm{BMIM}^{+} \mathrm{Cl}^{-}$. The separation factors, expressed by $\alpha=k_{1}{ }^{\prime} / k_{2}{ }^{\prime}$ for the neighboring 


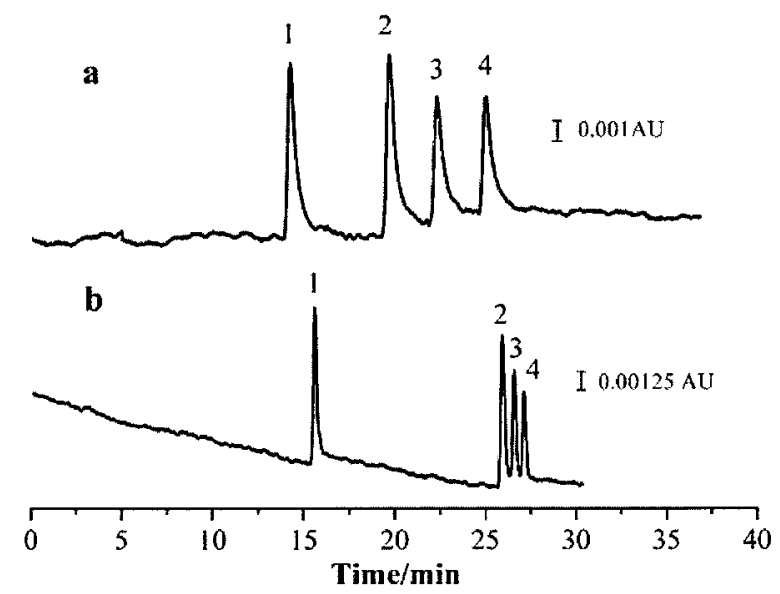

Fig. 2 Chromatograms of $2.5 \mathrm{mM}$ phenol derivatives with a mobile phase of $1.0 \mathrm{M} \mathrm{BMIM}^{+} \mathrm{Cl}^{-}$containing different concentrations of PVP: (a) 0.01 , (b) $0.1 \mathrm{M}$. Other chromatographic conditions are the same as Fig. 1. Peaks: (1) 2-naphthol, (2) phenol, (3) 4-chlorophenol, (4) 4-nitrophenol.

Table 1 Separation factors and theoretical plate numbers at the chromatogram of a mixture of 2-naphthol, phenol, 4-chlorophenol, and 4-nitrophenol under different mobile phase compositions

\begin{tabular}{lcc}
\hline \multirow{2}{*}{ Compound } & $\begin{array}{c}\text { Separation factor, } \\
\alpha^{\mathrm{a}}\end{array}$ & $\begin{array}{c}\text { Theoretical plate number, } \\
N^{\mathrm{b}}\end{array}$ \\
\hline 2-Naphthol & $2.50^{\mathrm{c}}$ & $332^{\mathrm{c}}$ \\
& $1.40^{\mathrm{d}}$ & $1204^{\mathrm{d}}$ \\
Phenol & $1.68^{\mathrm{e}}$ & $6320^{\mathrm{e}}$ \\
& $1.17^{\mathrm{c}}$ & $757^{\mathrm{c}}$ \\
4-Chlorophenol & $1.14^{\mathrm{d}}$ & $2281^{\mathrm{d}}$ \\
& $1.04^{\mathrm{e}}$ & $22907^{\mathrm{e}}$ \\
4-Nitrophenol & $1.28^{\mathrm{c}}$ & $1003^{\mathrm{c}}$ \\
& $1.14^{\mathrm{d}}$ & $4039^{\mathrm{d}}$ \\
& $1.03^{\mathrm{e}}$ & $63465^{\mathrm{e}}$ \\
& - & $2460^{\mathrm{c}}$ \\
& - & $3505^{\mathrm{d}}$ \\
\hline
\end{tabular}

a. $\alpha=k_{2}{ }^{\prime} k_{1}{ }^{\prime}$.

b. $N=16\left(t_{\mathrm{R}} / w_{\mathrm{R}}\right)^{2}$.

$\mathrm{c}-\mathrm{e}$. The concentrations of PVP in mobile phase: c, $0 \mathrm{M}$; d, $0.01 \mathrm{M}$; e $0.10 \mathrm{M}$ with $1.0 \mathrm{M} \mathrm{BMIM}^{+} \mathrm{Cl}^{-}$.

eluted compounds, were 2.50, 1.17, and 1.28 from 2-naphthol to 4-nitrophenol, where $k^{\prime}$ denotes the capacity factor, and is given by

$$
k^{\prime}=\left(t_{\mathrm{R}}-t_{0}\right) / t_{0}
$$

at a retention time $t_{\mathrm{R}}$ of a compound and at $t_{0}$ of the void volume, $v_{0}\left(=t_{0}\right)$, of the capillary tube. The theoretical plate numbers, calculated by $N=16\left(t_{\mathrm{R}} / w_{\mathrm{R}}\right)^{2}$, were 332, 757, 1003, and 2460 for 2-naphthol, phenol, 4-chlorophenol, and 4-nitrophenol, respectively (Fig. 1c), where $w_{\mathrm{R}}$ is the base width of a chromatogram of the compound.

The peaks appearing in Fig. 1c, however, were tailed by a little because the micro-solvent clusters did not so strongly assemble. To stabilize the ionic solvent clusters in the mixed solvent, a neutral polymer, PVP, was added to the mobile phase. Since ionic liquid molecules interact with polymers strongly by the hydrophobic interaction, ${ }^{20,21}$ it is expected that the ionic liquid

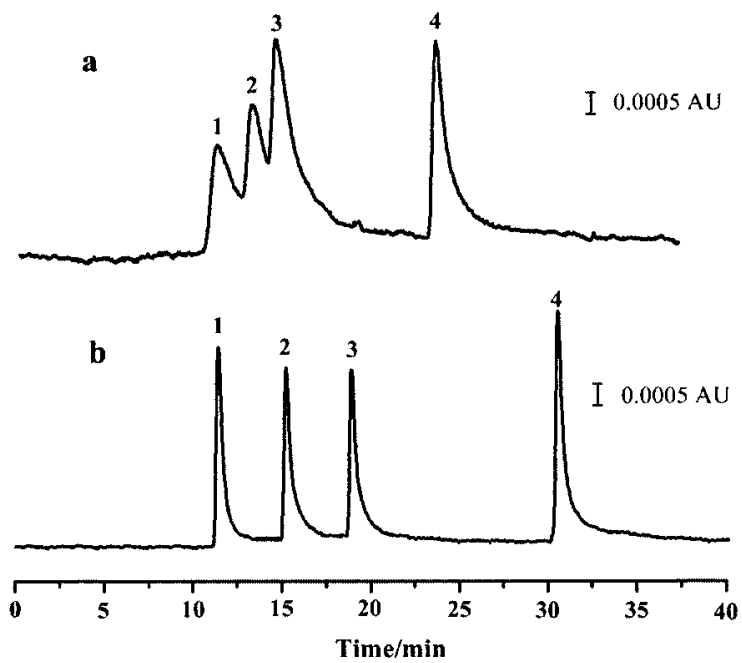

Fig. 3 Chromatograms of $2.5 \mathrm{mM}$ phenol derivatives with a mobile phase containing (a) $0.5 \mathrm{M} \mathrm{BMIM}^{+} \mathrm{Cl}^{-}$and $0.01 \mathrm{M} \mathrm{PVP}$, and (b) 0.8 $\mathrm{M} \mathrm{BMIM}{ }^{+} \mathrm{Cl}^{-}$and $0.01 \mathrm{M}$ PVP. Other chromatographic conditions are the same as Fig. 1. Peaks: (1) 2-naphthol, (2) phenol, (3) 4chlorophenol, (4) 1,3,5-naphthalenetrisulfonic acid.

molecules, $\mathrm{BMIM}^{+} \mathrm{Cl}^{-}$solvate to the polymer, leading to the growth of ionic liquid solvent clusters in the mixed solvents. At a concentration of $0.01 \mathrm{M}$ of PVP, phenols were not separated sufficiently, but at a concentration of $0.10 \mathrm{M}$ of PVP, phenols were separated well and sharp peaks were observed (Fig. 2).

PVP stabilizes the micro-solvent clusters, and the separation was significantly improved. Table 1 summarizes the separation factors and the theoretical plate numbers at different concentrations of PVP with $1.0 \mathrm{M}$ of $\mathrm{BMIM}^{+} \mathrm{Cl}^{-}$. The separation factors were larger than 1 for all of the neighboring compounds, and the theoretical plate numbers of the phenol derivatives were 6320, 22907, 63645, and 37184 for 2-naphthol, phenol, 4-chlorophenol, and 4-nitrophenol, respectively, under the conditions of $1.0 \mathrm{M}$ of $\mathrm{BMIM}^{+} \mathrm{Cl}^{-}, 0.1 \mathrm{M}$ of PVP, and a flow rate of $1 \mu \mathrm{L} \mathrm{min}{ }^{-1}$. The theoretical plate numbers are comparable to or much higher than HPLC. ${ }^{1,2}$

In the present method, both the ionic liquid and PVP are essential components for the separation of organic compounds, but the concentration of ionic liquid is more important than PVP to obtain a sufficient separation of the compounds.

Figure 3 shows the effects of $\mathrm{BMIM}^{+} \mathrm{Cl}^{-}$on the separation of organic compounds. The lower concentration of $\mathrm{BMIM}^{+} \mathrm{Cl}^{-}$ could not separate the compounds, and a concentration of $\mathrm{BMIM}^{+} \mathrm{Cl}^{-}$higher than $0.8 \mathrm{M}$ was required. Mixed solvents of water and the ionic liquid separated the organic compounds without a specific separation column, and PVP improved the separation, especially the theoretical plate numbers (Table 1). To know the effect of PVP in more detail for the separation of phenols, the capacity factor $\left(k^{\prime}\right)$ and the height equivalent theoretical plate, HETP, defined by HETP $=L / N$ of each compound, was plotted against the concentration of PVP (Fig. 4), where $L$ is capillary length $(450000 \mu \mathrm{m})$.

The capacity factors, $k^{\prime}$, of the analytes, especially phenol, 4chlorophenol, and 4-nitrophenol, increased at higher concentration of PVP (Fig. 4a), and the HETP values decreased with an increase of PVP from 0.01 to $0.1 \mathrm{M}$, and was then constant at higher than $0.10 \mathrm{M}$. The decreased HETP and the increased $k^{\prime}$ values suggest the growth of ionic liquid solvent clusters around PVP. 

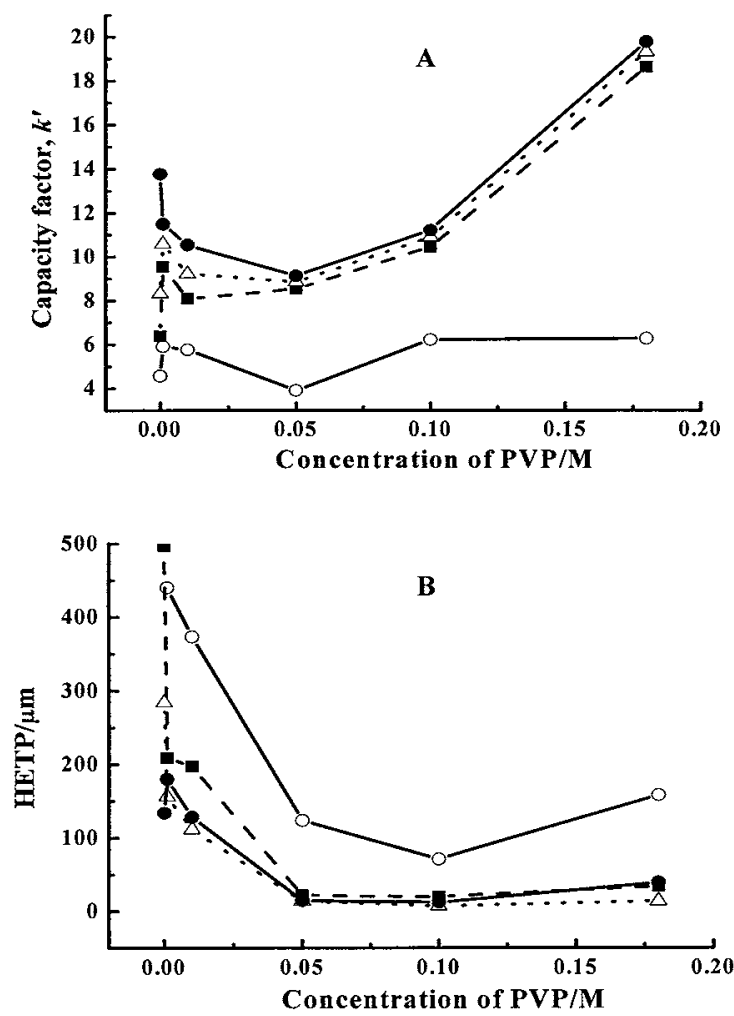

Fig. 4 Effects of the PVP concentrations on the capacity factor $\left(k^{\prime}\right)$ (a), and height equivalent theoretical plate (HETP) (b) of phenol derivatives at $1.0 \mathrm{M} \mathrm{BMIM}^{+} \mathrm{Cl}^{-}$. Other chromatographic conditions are the same as Fig. 1. O, 2-Naphthol; $\mathbf{m}$, phenol; $\triangle$, 4-chlorophenol; -, 4-nitrophenol.

Structural evidence for micro-phase separation in $\mathrm{BMIM}^{+} \mathrm{BF}_{4}-1$ water mixtures

A LAXS measurement was carried out for a mixture of water and $\mathrm{BMIM}^{+} \mathrm{BF}_{4}^{-}$instead of $\mathrm{BMIM}^{+} \mathrm{Cl}^{-}$, because $\mathrm{BMIM}^{+} \mathrm{Cl}^{-}$is solid at room temperature, and we cannot obtain the structural function of the pure liquid of $\mathrm{BMIM}^{+} \mathrm{Cl}^{-}$. We, of course, examined the effects of $\mathrm{BMIM}^{+} \mathrm{BF}_{4}{ }^{-}$on the separation of 2naththol and 4-nitrophenol. $\mathrm{BMIM}^{+} \mathrm{BF}_{4}^{-}$gave a similar separation behavior to $\mathrm{BMIM}^{+} \mathrm{Cl}^{-}$. The effects of anions of ionic liquids on the separation will be reported later.

The observed structure functions, $i(s) \mathrm{s}$ for the $\mathrm{BMIM}^{+} \mathrm{BF}_{4}-/$ water mixtures, are shown in Fig. 5 at different volume ratios $(0$, $3 / 7,5 / 5,7 / 3$, and 1.0), and the corresponding $g(r)$ s obtained from Fourier transformations of the $i(s) s$ are given in Fig. 6a. We note that $g(r)$ in the range of $r<2.5 \AA$ shows two sharp peaks at around 1.4 and $2.3 \AA$ for mixing ratios of $3 / 7,5 / 5,7 / 3$ and 1 , which are ascribed to intra-molecular interactions of $\mathrm{BMIM}^{+}$and $\mathrm{BF}_{4}^{-}$. The former peak at $1.4 \AA$ corresponds to the $\mathrm{C}-\mathrm{C}$ and $\mathrm{C}-\mathrm{N}$ bonds within $\mathrm{BMIM}^{+}$and the $\mathrm{B}-\mathrm{F}$ within $\mathrm{BF}_{4}{ }^{-}$and the latter at $2.3 \AA$ to the nonbonding $\mathrm{C} \cdots \mathrm{C}, \mathrm{C} \cdots \mathrm{N}$ interactions within $\mathrm{BMIM}^{+}$and the $\mathrm{F} \ldots \mathrm{F}$ within $\mathrm{BF}_{4}^{-}$. The $g(r)$ in neat water shows peaks at 2.8 and $4.7 \AA$, originating from the hydrogenbonding $\mathrm{O} \cdots \mathrm{O}$ interaction and the second-neighbor water-water interaction, respectively; both intensities decreased with the $\mathrm{BMIM}^{+} \mathrm{BF}_{4}^{-}$content. With regard to $g(r)$ for the $\mathrm{BMIM}^{+} \mathrm{BF}_{4}^{-}$water system, the intra- and inter-molecular interactions are overlapped at around the range of $2.5<r<6 \AA$ due to the relatively large molecular size and complicated structure of $\mathrm{BMIM}^{+}$. Thus, we extracted the inter-molecular interaction, $g_{\text {inter }}(r)$, by subtracting the intra-molecular interaction, $g_{\text {intra }}(r)$,

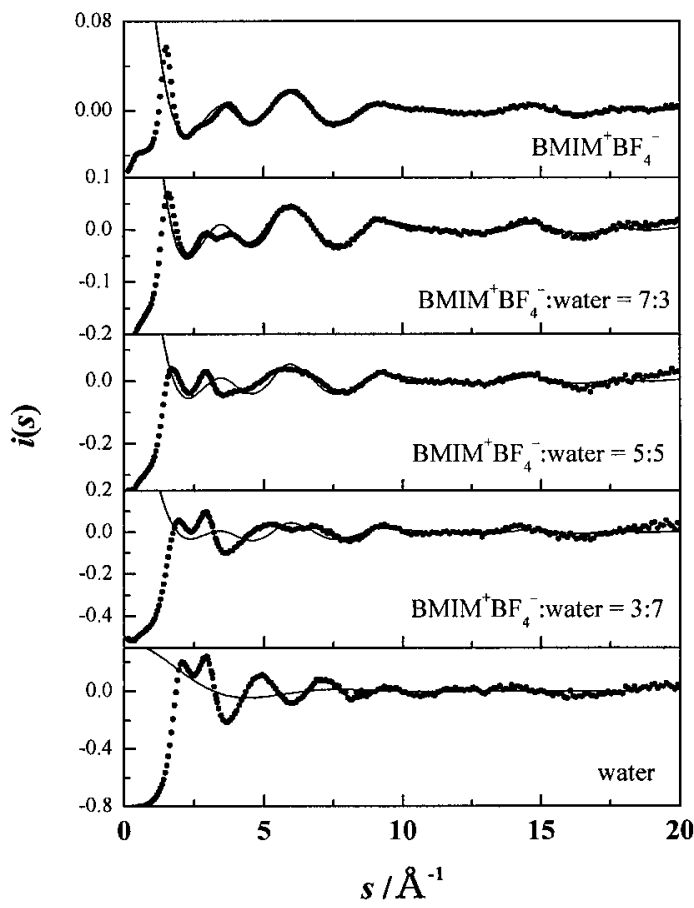

Fig. 5 Structure functions, $i(s)$, observed (dotted line) and calculated using crystal data (solid line) for the $\mathrm{BMIM}^{+} \mathrm{BF}^{-}$water mixtures.

from the observed $g(r)$.

The observed $g(r)$ is given as $g(r)=g_{\text {intra }}(r)+g_{\text {inter }}(r) ; g_{\text {intra }}(r)$ is evaluated from known structure parameters of $\mathrm{BMIM}^{+}, \mathrm{BF}_{4}^{-}$and water in each crystal, ${ }^{22,23}$ as follows:

$$
g_{\text {intra }}(r)=\alpha \cdot g_{\text {intra, } \mathrm{BMIM}}(r)+\beta \cdot g_{\text {intra, } \mathrm{BF} 4}(r)+\chi \cdot g_{\text {intra }, \mathrm{H} 2 \mathrm{O}}(r),
$$

where $\alpha, \beta$ and $\chi$ denote the mole fraction of the corresponding molecule in the $\mathrm{BMIM}^{+} \mathrm{BF}_{4}{ }^{-} /$water mixture examined. It has already been established that $\mathrm{BMIM}^{+}$in $\mathrm{BMIM}^{+} \mathrm{BF}_{4}^{-}$and/or other ionic liquids involves two conformers with respect to the alkyl group of $\mathrm{BMIM}^{+}$(trans-trans and gauche-trans $\mathrm{BMIM}^{+}$) in equilibrium, and the two conformers almost equally exist in neat ionic liquids. ${ }^{24-26}$ The conformer ratio in the $\mathrm{BMIM}^{+} \mathrm{BF}_{4}-/$ water system, however, changes with the mixing ratio of the mixed solvents, ${ }^{27}$ but the equilibrium constants between the conformers are not known yet. Thus, it is difficult to obtain the correct value of $g_{\text {intra }}(r)$ for $\mathrm{BMIM}^{+}$in binary mixtures. Therefore, we assumed that the distribution of the trans-trans and gauche-trans $\mathrm{BMIM}^{+}$is comparable, that is, $g_{\text {intra,BMIM }}(r)=$ $0.5 g_{\text {intra,trans-trans }}(r)+0.5 g_{\text {intra,trans-gauch }}(r)$, as a first approximation. Indeed, the intra-molecular $i_{\text {intra }}(s)$ calculated by using the $g_{\text {intra }}(r)$ (solid line) obtained from Eq. (3) well reproduces the observed $i(s)$ (dotted line) at the high $s$ values corresponding to the intramolecular interaction for all of the mixing ratios as given in Fig. 5.

The $g_{\text {inter }}(r)$ value obtained for the $\mathrm{BMIM}^{+} \mathrm{BF}_{4}-/$ water system at $1 \AA<r<8 \AA$ is shown in Fig. 6b. The $g_{\text {inter }}(r)$ value is almost zero in the range of $r<2 \AA$ for all samples, indicating that the intra-molecular interactions were subtracted correctly. The peaks at $2.8,4.5$ and $7 \AA$ for pure water were weaken with increasing $\mathrm{BMIM}^{+} \mathrm{BF}_{4}^{-}$, and that at $5.5 \AA$ were strengthened. It should be noted that the isosbestic points were observed at 4.9 and $6.2 \AA$ in Fig. 6(b), implying that the liquid structures of neat water and $\mathrm{BMIM}^{+} \mathrm{BF}_{4}^{-}$exist in the $\mathrm{BMIM}^{+} \mathrm{BF}_{4}-$ /water mixtures; namely, solvent clusters of water and $\mathrm{BMIM}^{+} \mathrm{BF}_{4}{ }^{-}$are formed in the mixture. The peak at $2.8 \AA$ (hydrogen bonding $\mathrm{O} \cdots \mathrm{O}$ 

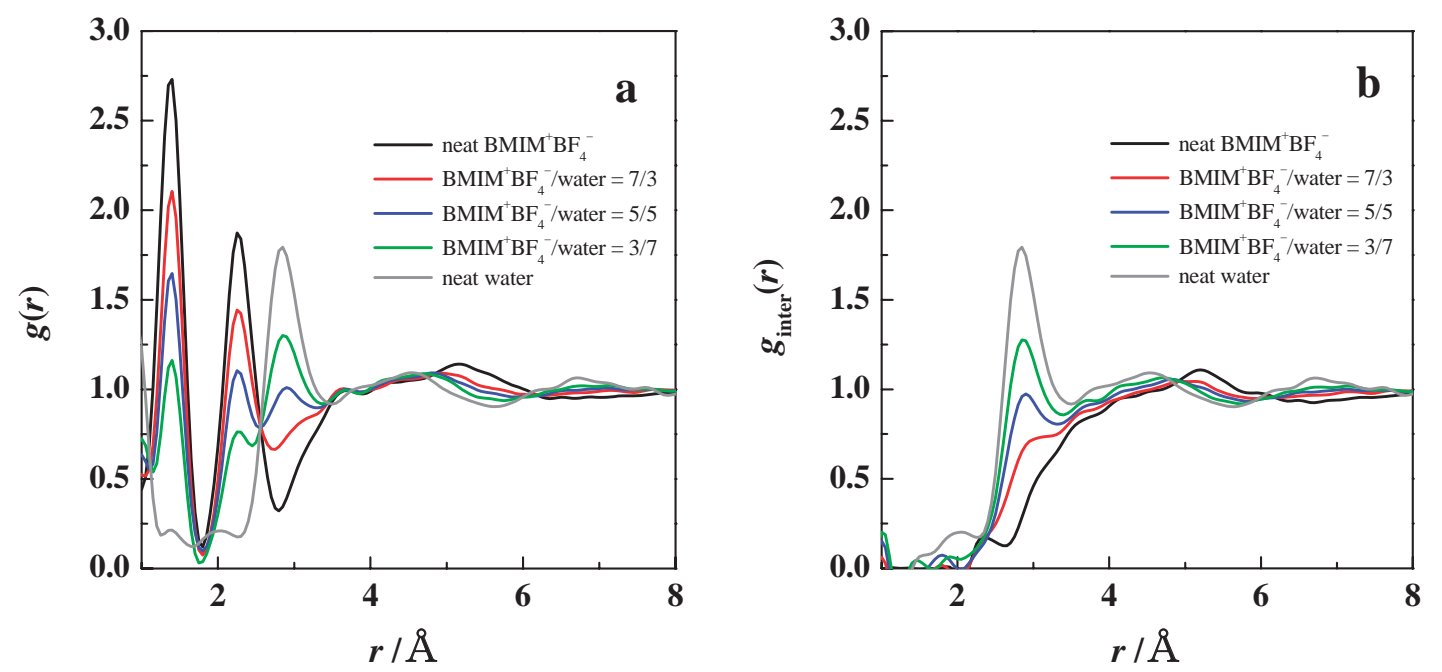

Fig. 6 Observed $g(r)$ (a) and $g_{\text {inter }}(r)$ calculated using crystal data of water, $\mathrm{BF}_{4}{ }^{-}$and two $\mathrm{BMIM}^{+}$ conformers (b) for the $\mathrm{BMIM}^{+} \mathrm{BF}_{4}-$ wwater mixtures.

interaction) for neat water is monotonically weakened with increasing $\mathrm{BMIM}^{+} \mathrm{BF}_{4}^{-}$.

Figure 7 shows the calculated $g_{\text {inter }}(r)$ on the basis of neat water and $\mathrm{BMIM}^{+} \mathrm{BF}_{4}^{-} g_{\text {inter }}(r) \mathrm{s}$, together with the observed one for the mixing ratio $\mathrm{v} / \mathrm{v}=5 / 5$. The calculated $g_{\text {inter }}(r)$ well reproduces the observed one, implying that the hydrogenbonding structure of neat water is maintained in the $\mathrm{BMIM}^{+} \mathrm{BF}_{4}^{-} /$ water mixture. This result also indicates a heterogeneous mixing or micro-phase separation of water and $\mathrm{BMIM}^{+} \mathrm{BF}_{4}^{-}$, like acetonitrile/water or various alcohol/water systems. ${ }^{9}$ Indeed, Katayanagi et al. reported based on a thermodynamic study for $\mathrm{BMIM}^{+} \mathrm{BF}_{4}^{-}$/water mixture that the hydration around $\mathrm{BMIM}^{+}$ and $\mathrm{BF}_{4}^{-}$ions is weak in the water-rich mixing ratio, and an aggregation among the ions occurs at the mixing mole fraction, $x_{\text {BMIMBF4 }}=0.015 .{ }^{28}$ In the $\mathrm{BMIM}^{+} \mathrm{BF}_{4}{ }^{-}$-rich region, the local cluster, such as neat $\mathrm{BMIM}^{+} \mathrm{BF}_{4}^{-}$, forms at $x_{\mathrm{BMIMBF}}>0.5 .^{28}$ Furthermore, an IR study of $\mathrm{BMIM}^{+} \mathrm{BF}_{4}{ }^{-} /$water mixture by Jeon et al. reported that the $\mathrm{BF}_{4}^{-}$can easily interact with the $\mathrm{BMIM}^{+}$, rather than the water at any mole fraction. ${ }^{27}$ These results are consistent with that obtained from our X-ray scattering for the water-rich mole fraction in the present work. We can thus conclude that $\mathrm{BMIM}^{+} \mathrm{BF}_{4}{ }^{-}$in the $\mathrm{BMIM}^{+} \mathrm{BF}_{4}-$ /water mixture forms clusters as in the neat state, which causes micro-phase separation involving $\mathrm{BMIM}^{+} \mathrm{BF}_{4}^{-}$and water clusters.

\section{Separation mechanism}

The LAXS data suggest the formation of micro-solvent cluster formation in the mixed solvent. Ueki et al. reported the occurrence of a phase separation of ionic liquid when biopolymers were dissolved in the ionic liquids, showing a cloud temperature. ${ }^{21}$ Thus, PVP enhances the aggregation of the ionic liquid around the polymer in aqueous mixed solvents. Aromatic compounds are concentrated in the ionic liquid clusters around PVP. On the other hand, polar or ionic compounds are concentrated in water clusters. Water molecules adhere to the inner surface of the capillary tube, and the water structure is strengthened through increased hydrogen bonding with high viscosity. ${ }^{29}$ Ionic compounds therefore elute slowly as 1,3,5-naphthalenetrisufonic acid shown in Fig. 3. The increased micro-solvent clusters of the ionic liquid increase the theoretical plate numbers (Table 1).

The mixture of water and $\mathrm{BMIM}^{+} \mathrm{Cl}^{-}$is not a homogeneous

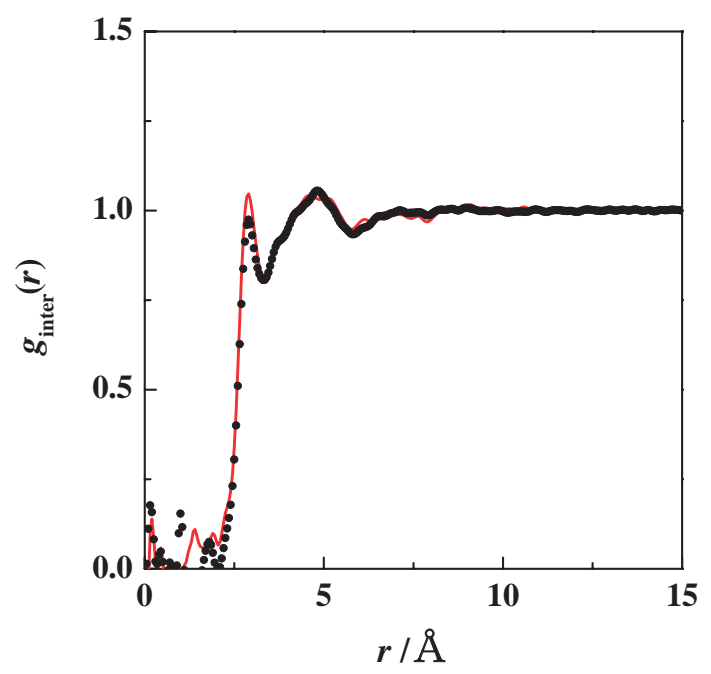

Fig. $7 g_{\text {inter }}(r)$ calculated on the basis of neat water and $\mathrm{BMIM}^{+} \mathrm{BF}_{4}^{-}$ for the mixing ratio $\mathrm{v} / \mathrm{v}=5 / 5$, together with the corresponding observed one (dotted line). The calculated $g(r)$ (red-solid line) is given as $g_{\text {calc }}(r)=\left(g_{\text {water }}(r)+g_{\text {BMIMBF } 4}(r)\right) / 2$.

mixed solvent on the micro-scale, and forms micro-solvent clusters of water and the ionic liquid. The micro-solvent clusters formed in the mixed solvents are strengthened upon the addition of a polymer, like PVP, in a capillary tube, and are localized to the center, while the water clusters are concentrated on the surface of the capillary tube. The injected compounds distribute between the organic solvent clusters around PVP at the center of the capillary tube and the water clusters at the surface of the capillary tube. The compounds distributed to water clusters move slowly. This is a schematic mechanism for the separation of compounds in a capillary tube with mixed solvents of $\mathrm{BMIM}^{+} \mathrm{Cl}^{-}$containing PVP during a flow of the injected sample (Scheme 1).

We propose here a new type of separation method based on "micro-solvent cluster extraction" using $\mathrm{BMIM}^{+} \mathrm{Cl}^{-}$and PVP in an aqueous solution in a capillary tube without a specific separation column. Other ionic liquids can also separate compounds by passing through a capillary tube. The research is in progress, and results will be reported elsewhere. 


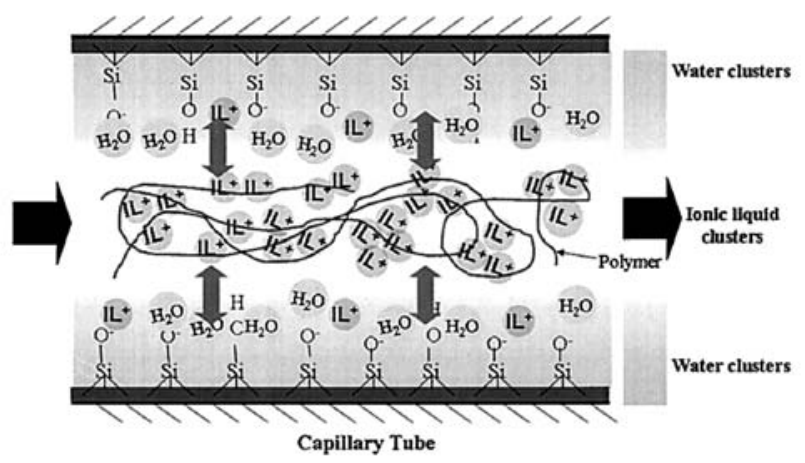

Scheme 1 Proposed mechanism for "micro-solvent cluster extraction" of compounds between $\mathrm{BMIM}^{+} \mathrm{Cl}^{-}$clusters and water clusters formed at the center and on the surface of a capillary tube, respectively.

\section{Acknowledgements}

The authors would like to acknowledge financial support through a Grand-in-Aid B, (No. 1935004) from The Ministry of Education, Culture, Sports, Science and Technology (MEXT), Japan and a Grant-in-Aid for Japan Science Promotion Society (JSPS) Fellows (No. 19003963).

\section{References}

1. G. A. Baker, S. N. Baker, S. Pandey, and F. V. Bright, Analyst [London], 2005, 130, 800.

2. S. A. Shamsi and N. D. Danielson, J. Sep. Sci., 2007, 30, 1729.

3. T. Takamuku, M. Tabata, A. Yamaguchi, J. Nishimoto, M. Kumamoto, H. Wakita, and T. Yamaguchi, J. Phys. Chem. $B, \mathbf{1 9 9 8}, 102,8880$.

4. Y. G. Wu, M. Tabata, and T. Takamuku, J. Mol. Liq., 2001, 94, 273.

5. Y. G. Wu, M. Tabata, and T. Takamuku, Talanta, 2001, 54, 69.

6. T. Takamuku, A. Yamaguchi, D. Matsuo, M. Tabata, M. Kumamoto, J. Nishimoto, K. Yoshida, T. Yamaguchi, M. Nagao, T. Otomo, and T. Adachi, J. Phys. Chem. B, 2001 , 105,6236 .

7. Y. G. Wu and M. Tabata, J. Solution Chem., 2004, 33, 777.

8. Y. G. Wu, M. Tabata, and T. Takamuku, J. Solution Chem., 2002, 31, 381.

9. T. Takamuku, A. Yamaguchi, D. Matsuo, M. Tabata, T.
Yamaguchi, T. Otomo, and T. Adachi, J. Phys. Chem. B, 2001, 105, 10101.

10. M. Tabata, Y. G. Wu, T. Charoenraks, and S. S. Samaratunga, Bull. Chem. Soc. Jpn., 2006, 79, 1742.

11. J. L. Anderson, D. W. Armstrong, and G. Wei, Anal. Chem., 2006, 78, 2892.

12. M. Isshiki, Y. Ohishi, S. Goto, K. Takeshita, and T. Ishikawa, Nucl. Instrum. Methods Phys. Res., Sect. A, 2001, $467-468,663$.

13. S. Kohara, K. Suzuya, Y. Kashihara, N. Matsumoto, N. Umesaki, and I. Sakai, Nucl. Instrum. Methods Phys. Res., Sect. A, 2001, 467 - 468, 1030.

14. S. Sasaki, KEK Report, 1991, 90-16, National Laboratory for High Energy Physics, Japan.

15. D. T. Cromer and J. B. Mann, J. Chem. Phys., 1967, 47, 1892.

16. K. Fujii, S. Shiro, S. Fukuda, T. Takamuku, S. Kohara, Y. Kameda, Y. Umebayashi, and S. Ishiguro, J. Mol. Liq., 2008, accepted.

17. E. N. Maslen, A. G. Fox, and M. A. O'Keefe, in "International Tables for Crystallography", 1999, Vol. C, Kluwer, Dorfrecht, 572.

18. G. Johanson and M. Sandstörm, Chem. Scr., 1973, 4, 195.

19. A. E. Martell and R. M. Smith, "Critical Stability Constants", 1977, Vol. 3, Other Organic Ligands, Plenum Press, New York and London.

20. T. Ueki and M. Watanabe, Langmuir, 2007, 23, 988.

21. T. Ueki, T. Karino, Y. Kobayashi, M. Shibayama, and M. Watanabe, J. Phys. Chem. B, 2007, 111, 4750.

22. J. D. Holbrey, W. M. Reichert, M. Nieuwenhuyzen, S. Johnston, K. R. Seddon, and R. D. Rogers, Chem. Commun., 2003, 1636.

23. A. R. Choudhury, N. Winterton, A. Steiner, A. I. Cooper, and K. A. Johnson, J. Am. Chem. Soc., 2005, 127, 16792.

24. H. Katayanagi, S. Hayashi, H. Hamaguchi, and K. Nishikawa, Chem. Phys. Lett., 2004, 392, 460.

25. J. N. A. C. Lopes and A. A. H. Padua, J. Phys. Chem. B, 2006, 110, 7485.

26. R. Ozawa, S. Hayashi, S. Saha, A. Kobayashi, and H. Hamaguchi, Chem. Lett., 2003, 32, 948.

27. Y. Jeon, J. Sung, D. Kim, C. Seo, H. Cheong, Y. Ouchi, R. Ozawa, and H. Hamaguchi, J. Phys. Chem. B, 2008, 112, 923.

28. H. Katayanagi, K. Nishikawa, H. Shimozaki, K. Miki, P. Westh, and Y. Koga, J. Phys. Chem. B, 2004, 108, 19451.

29. T. Tsukahara, A. Hibara, Y. Ikeda, and T. Kitamori, Angew. Chem., Int. Ed., 2007, 46, 1180. 